\title{
Erratum: Quantum Thermodynamics of Correlated-Catalytic State Conversion at Small Scale [Phys. Rev. Lett. 126, 150502 (2021)]
}

\author{
Naoto Shiraishi and Takahiro Sagawa
}

(Received 29 January 2022; published 23 February 2022)

DOI: 10.1103/PhysRevLett.128.089901

Theorem 3 of this Letter states that

Consider four quantum states $\rho, \rho^{\prime}, \eta$, and $\eta^{\prime}$ of the system $S$. Then, $S(\rho \| \eta) \geq S\left(\rho^{\prime} \| \eta^{\prime}\right)$ holds if and only if there exists a catalyst $C$ and its two states $c, d$, and a CPTP map which converts $\eta \otimes d$ to $\eta^{\prime} \otimes d$ and $\rho \otimes c$ to $\tau$ satisfying (i) $\operatorname{Tr}_{S}[\tau]=c$, (ii) $\operatorname{Tr}_{C}[\tau]$ is arbitrarily close to $\rho^{\prime}$, (iii) the correlation between $S$ and $C$ in $\tau$ is arbitrarily small.

In this theorem, the following additional condition $\mathrm{C}$ on catalysts was missing: (C) The support of $d$ contains that of $c$; $\operatorname{supp}(c) \subset \operatorname{supp}(d)$. Without this condition $\mathrm{C}, S_{1}(c \| d)$ diverges, and the transformation in (S.1) in the Supplemental Material of this Letter no longer holds [1]. Moreover, without condition C, any state transformation becomes possible, as pointed out by Rubboli and Tomamichel (footnote 3 of Ref. [2]). We note, however, that the entire argument of this Letter remains correct only by adding condition $\mathrm{C}$ to the statement of Theorem 3 . We also note that this modification only touches the definition of catalytic state conversions, and therefore Theorem 3 still applies to any four quantum states $\rho, \rho^{\prime}, \eta$, and $\eta^{\prime}$ of the system $S$. The correct full statement of Theorem 3 is the following:

Consider four quantum states $\rho, \rho^{\prime}, \eta$, and $\eta^{\prime}$ of the system $S$. Then, $S(\rho \| \eta) \geq S\left(\rho^{\prime} \| \eta^{\prime}\right)$ holds if and only if there exists a catalyst $C$ and its two states $c, d$ satisfying $\operatorname{supp}(c) \subset \operatorname{supp}(d)$, and a CPTP map which converts $\eta \otimes d$ to $\eta^{\prime} \otimes d$ and $\rho \otimes c$ to $\tau$ satisfying $(i) \operatorname{Tr}_{S}[\tau]=c$, (ii) $\operatorname{Tr}_{C}[\tau]$ is arbitrarily close to $\rho^{\prime}$, (iii) the correlation between $S$ and $C$ in $\tau$ is arbitrarily small.

A similar correction is needed for the description of Theorem 3 in the Supplemental Material [1].

[1] N. Shiraishi and T. Sagawa, Quantum thermodynamics of correlated-catalytic state conversion at small-scale, Phys. Rev. Lett. 126, 150502 (2021).

[2] R. Rubboli and M. Tomamichel, Fundamental limits on correlated catalytic state transformations, arXiv:2111.13356. 